\title{
A Delay-tolerant MAC Protocol for Emergency Care in WBAN Considering Preemptive and Non- preemptive Methods
}

\author{
Shah Murtaza Rashid Al Masud, Aloke Kumar Saha \\ Department of Computer Science and Engineering \\ University of Asia Pacific, Dhaka, Bangladesh
}

\begin{abstract}
For facilitating pilgrims with no delay, quick and real-time emergency medical services at ritual sites a delaytolerant Medium Access Control (MAC) protocol for the IEEE 802.15.6 standard based Wireless Body Area Networks (WBANs) has been proposed. Since MAC protocol is application-specific hence any particular MAC technique may not be appropriate for diverse applications. In this research work, we consider dealing with medical emergency traffics which is random, independent of each other and can be generated at any time. Moreover, emergency traffics must be transmitted ahead of normal medical data or emergency traffic with a lower severity level; because any delay in emergency data transmission may endanger patients' life. The proposed MAC protocol is compared with both preemptive and non-preemptive methods. Where, a modified MAC superframe (SF) structure, minimum backoff period and minimum Contention Window (CWmin) for quick data access to the IEEE 802.15.6 standard based EAP channel are also considered. The proposed delay-tolerant MAC protocol has been experimented with and simulated by the Castalia simulator which is based on the OMNeT++ platform. The experimental results show that data transmission using the preemptive method works faster with reduced delay than that of the non-preemptive method. Furthermore, the delay metric of the proposed delaytolerant MAC protocol is analyzed, calculated and compared with the current Traffic-aware TA-MAC protocol. Results demonstrate that delay is relatively low during emergency data transmission using the proposed MAC in WBANs environment.
\end{abstract}

Keywords-WBAN; MAC; preemptive; non-preemptive; delay; emergency traffic

\section{INTRODUCTION}

Due to the huge medical concerns of pilgrimage at overcrowded Hajj ritual sites in Makkah and Madinah and Kumbh Mela in India, World Health Organization (WHO), Ministry of Health (MoH) of Saudi Government, and Government of India at different times provided with medical awareness guidelines for the pilgrims. During pilgrimage it is utmost important to identify pilgrims with serious medical issues and to provide them with adequate healthcare services [1-2]. In addition, a very few pilgrims' monitoring and healthcare technologies have been evolved including GPS, RFID, and WSN based ITS [3-7]. However, existing healthcare facilities are rarely able to observe urgent medical issues in an immediate and speedy way. Therefore, there is a vital need of emergent healthcare technology to abridge pilgrims' emergency medical problems.
According to the study [8-9], WBANs is an embryonic technology consists of numerous body sensors and a body coordinator and can be a greater option for medical applications at different health conditions. WBAN can deal with diverse traffic types including emergency, on-demand and normal traffic. In healthcare applications patients' data must be transferred ahead of other non-medical and low critical medical data because any data lost or delay may endanger the life. Among the WBANs heterogeneous traffics, emergency traffic is very unpredictable in nature. Emergency traffic can be produced in both regular and random manner. Generally, emergency traffics need to transfer in Contention-Free Phase (CFP) and non-scheduled mode which is opposite to normal medical traffic that can be sent in scheduled phase and in Contention Access Phase (CAP). However, the problem may occur during transmission of multiple emergency data concurrently that may result in inefficient transmission of medical data with severe delay, data lost and re-transmission, collision and excessive energy consumption.

Since, WBAN is energy and delay sensitive, hence, one particular communication technology and Medium Access Control (MAC) protocol will hardly be suitable for every possible WBAN applications. The IEEE802.15.6 standard based MAC protocol is anticipated to handle heterogeneous traffics where Exclusive Access Phase I and II (EAP I and II) is designed for emergency data access, Random Access Phase I and II (RAP) for on-demand traffics, and Managed Access Phase I and II (MAP) for normal medical traffics. But, EAP I and EAP II of MAC superframe (SF) structure work based on Contention Access Phase (CAP). CAP leads data traffic to contend with each other if multiple data aggregate at coordinator needs to simultaneously access the channel, which is the main reason for data collision and data loss which result in higher delay and excessive power consumption. Hence, there is a need for deploying appropriate priority and queue model for designing MAC protocol that should ensure high priority data to be given higher priority during transmission. The existing MAC superframe may not be suitable for critical data management for medical applications considering both IEEE 802.15.6 and IEEE 802.15.4 standards [10]. According to their research, for handling medical emergencies, CFP is proposed. But, to the best of our knowledge, emergency physiological data can be generated and transmitted at any time; hence, time-bounded scheme and technology may not be suitable for medical emergency applications. Moreover, unlike 
the IEEE802.15.6 standard, the IEEE802.15.4 standard does not support data classification and prioritization features. Therefore, the IEEE 802.15.4 MAC superframe is also not suitable to be used for monitoring pilgrims' emergencies and critical health issues during Hajj, Kumbh Mela or any overcrowded event.

To tackle medical emergencies using WBANs, in our research we proposed delay-tolerant MAC protocol. For deploying delay efficient MAC for WBAN we primarily considered both preemptive and non-preemptive methods to effectively transmit pilgrims' data to the healthcare station. In non-preemptive method, packets that are in undergoing services are allowed to finish services first without disturbance even in the meantime if packets with higher priority arrive. Besides, in the non-preemptive method, the packet with the highest priority enters service first only when the server becomes idle or free. Thus, the non-preemptive priority model is not able to deliver medical data with a higher priority before data with a lower priority level, which results in higher delay. Hence, for tackling medical emergency situation, we finally considered preemptive method for developing delay-tolerant MAC protocol, because in this scheme, data with higher priority must access the channel or SF timeslot ahead of lower priority medical data thus results in lower delay in emergency data transmission with higher severity.

To experiment, analyze and validate the obtained results, the proposed delay-tolerant MAC protocol has been simulated with Castalia simulator which is based on OMNeT++ simulator. The result is analyzed considering both preemptive and non-preemptive methods. Moreover, the delay metric of the proposed delay-tolerant MAC protocol is compared with up-to-date Traffic-aware MAC protocol (TA-MAC). Results exhibit that delay is comparatively very low during transmission of emergency data with different severity levels using preemptive method in WBANs environment due to less queuing delay, no data re-transmission and no collision.

The rest of the research paper is structured as follows: Section 2 presents the related work. Classification of traffics, proposed modified MAC superframe structure, algorithms and network management procedures are explained in Section 3. Results are discussed in Section 4. Finally, the paper ends with conclusion in Section 5.

\section{RELATED WORK}

This section explores the literature in order to discover the existing MAC methods and techniques and their limitations that are already being designed and deployed for WBANs healthcare applications considering different Quality of Service (QoS) issues. The delay tolerant MAC protocol must consider WBANs heterogeneous traffic. The QoS proficient MAC protocol must consider diverse system requirements and network development challenges. Major challenges and requirements include data classification and prioritization, energy consumption issues of sensor nodes, delay in transmission, data rate and timely delivery of medical data. In WBANs applications, any loss of physiological data and excessive delay in transmission may jeopardize patients' life.
Considering WBANs MAC requirements and other environmental issues some researchers have designed and proposed several priority and QoS efficient MAC protocols for WBAN. An energy efficient Adaptive (A-MAC) MAC protocol has been proposed in [11]. In this IEEE 802.15.6 based MAC protocol, data are classified into three priority classes and an improved MAC superframe has also been proposed. The existing superframe is restructured into four different periods or phase such as beacon phase, aperiodic contention access phase, periodically scheduled phase or contention-free phase, and an inactive phase. The slots lengths (time duration) for access phases are being adjusted according to the priority level of data, moreover, body sensors must compete for accessing channel according to the channel access mechanism.

A radio wake-up mechanism based MAC protocol for WBAN is proposed in [12]. Authors consider data classification and prioritization to achieve the goal, which is 'prolong the network lifetime'. The protocol is designed according to the IEEE802.15.4 standard, where the superframe is also modified and improved. To represent the limited capacity of the buffers, an asymmetric hidden Markov model is also illustrated. Another MAC protocol that has been proposed by the authors [10] where emergency traffics are being classified into diverse data severity levels based on the threshold values. In addition, authors have suggested for the modification of the MAC superframe based on different time slots for accessing communication channel. To access the different channel, data is further divided into high priority and low priority data. Though data delay and throughput are presented in the result, however, energy consumption issue is not defined. Moreover, no direction is provided to handle various emergency data.

The authors [13] proposed IEEE802.15.4 based TA-MAC protocol of diverse phases of CAP and different levels of traffic priority. In the CAP, traffic-aware MAC utilizes the prioritybased CSMA/CA procedure that is supported by the IEEE802.15.6 standard to satisfy WBANs standard; however, the protocol is restricted to IEEE802.15.4 standard. In [14], the IEEE 802.15.6 Traffic Priority based Channel Assignment Technique (TP-CAT) has been projected. Authors have recommended adaptive time slot management algorithms based on data threshold values for QoS efficient TP-CAT.

A novel Energy Efficient and Load Balanced Priority Queue Algorithm (ELBPQA) has been proposed [15], where, traffic criticality is defined by data priority levels such as lowmedium-high priority data, and thus data are scheduled and transmitted. Data priority and modified superframe are proposed for the IEEE802.15.4 standard, and besides, CSMA/CA mechanism is used to tackle data with different priorities of energy-efficient MAC protocol [16]. A Markov model has also been proposed in order to identify the state of WBANs sensor nodes.

Authors [17] have proposed Traffic Adaptive Priority (TAP-MAC) MAC protocol with a revised MAC superframe structure. The goal of TAP-MAC is to reduce collisions and data re-transmission that results in lower delay and minimal energy consumption. Naturally, during CAP, low-priority 
traffic cannot dominant over high-priority traffic thus results in lower throughput, higher delay and high energy consumption in WBAN [17]. The existing standard for data communication does not offer a differentiated QoS to the diverse traffic therefore, a Priority-based Adaptive (PA) MAC protocol for WBAN was proposed in [18]. In PA-MAC, data is classified into four different types. In this protocol, according to data priority level, MAC superframe timeslots are being dynamically allocated. The proposed protocol works based on well-designed IEEE 802.15.4 and IEEE 802.15.6 standards. The PA-MAC protocol cares more on traffics with higher priority than that of lower priority which affects the overall network quality.

The above mentioned MAC protocols have been proposed and designed on the basis of the IEEE 802.15.4 and the IEEE 802.15.6 standards. In WBANs communication, generally, higher-priority traffic is generally the lowermost loaded traffic and lower-priority traffic is typically the highermost loaded traffic, hence MAC protocols need to accept either one or both of the aforementioned concepts in order to ignore the harmful situation during data transmission considering diverse QoS including delay and energy consumption issues. In addition, there is an essential requisite to deliver and provide services with utmost importance to manage emergency medical packets and its severity levels. Hence, there is an utmost necessity to design and develop MAC protocol for WBAN that should deal with various QoS related issues along with the traffic-severity and priority of WBAN applications for real-time and quick monitoring of patients at overcrowded environment.

\section{DELAY-TOLERANT MAC PROTOCOL FOR WBAN}

\section{A. Data Classification and Determination of Severity Levels}

For WBANs coordinator it is less challenging to tackle one or a few emergency event than multiple emergency events simultaneously. To build a delay-tolerant MAC protocol for WBAN application, we classify the emergency traffic into six different categories based on the level of severity, which is presented in Table I. Data severity or criticality is basically meant by a level of medical urgency or emergency. Here in this research, we classified, ordered, organized, prioritized WBAN emergency traffic based on their QoS requisites for MAC which is necessitated for a novel solution in WBAN applications.

Emergency data is event triggered and relies on lifethreatening situation to be generated. Hence, emergency data requires smooth and quick transmission in WBAN medium in an efficient way.

TABLE I. SEVERITY LEVEL OF DISEASES

\begin{tabular}{|l|l|}
\hline Medical Syndromes/Diseases & Level of Severity \\
\hline Respiratory Syndromes & Extremely severe traffic \\
\hline Cardiovascular Problems & Very high severe traffic \\
\hline Diabetes Mellitus & High severe traffic \\
\hline Blood Pressure (BP) & Moderately severe traffic \\
\hline Gastroenteritis & Low severe traffic \\
\hline Body Temperature (BT) & Very low severe traffic \\
\hline
\end{tabular}

\section{B. Modified MAC Superframe for Emergency Data} Transmission

Based on the nature of WBAN operations and applications, the IEEE 802.15.6 and IEEE 802.15.4 based MAC superframe can be re-structured in to different time frame [8], where, beacon period of the same length encircles the entire timeslot of the superframe. Using the IEEE 802.15.6 standard and its associated MAC protocols, WBANs are supposed to transmit data in three different modes to access channels enabling beacon mode and non-beacon mode. Beacon mode supports MAC with superframe structure; on the other hand, non-beacon mode supports MAC with superframe and without superframe structure to operate in WBAN environment. The superframe is divided into two phases, such as aperiodic CAP and periodic scheduled access phase. CAP is non-scheduled and aperiodic in nature; moreover, CAP is random and dynamic. In IEEE 802.15.6 based CAP supports EAP 1 and EAP 2. It also supports RAP 1 and RAP 2 and the CAP slot itself. However, MAP is scheduled based access phase which is supported by CFP. The slots of superframe have different periods or duration, and lengths are identified based on the number of timeslots. Hence the MAC superframe structure can be improved and restructured by neutralizing definite time periods. In our research, we modify and update the existing MAC superframe in order to avoid data collision during simultaneous transmission of emergency medical data. The proposed MAC superframe structure is presented in Fig. 1.

\begin{tabular}{|c|c|c|c|c|}
\hline Beacon & $\begin{array}{c}\text { UP7 } \\
\text { CSMA/CA }\end{array}$ & $\begin{array}{c}\text { All UPs } \\
\text { CSMA/CA }\end{array}$ & $\begin{array}{c}\text { Polling } \\
\text { Mechanism }\end{array}$ & Beacon \\
\hline \multirow[t]{2}{*}{ B } & EAP & RAP & MAP & \multirow[t]{2}{*}{ B } \\
\hline & $\begin{array}{l}\mathrm{T}_{\text {EAp }} \text { belong to } \\
\text { Emergency } \\
\text { medical data }\end{array}$ & $\begin{array}{l}T_{\text {RAp belong to }} \\
\text { On-demand data }\end{array}$ & $\begin{array}{l}\mathrm{T}_{\mathrm{MAA}} \text { belong to } \\
\text { Normal medical } \\
\text { data }\end{array}$ & \\
\hline \multicolumn{5}{|c|}{ Beacon Period (Superframe) } \\
\hline $\begin{array}{l}\text { EAP: ex } \\
\text { RAP: rar } \\
\text { MAP: } m\end{array}$ & $\begin{array}{l}\text { usive access ph } \\
\text { dom access phas } \\
\text { naged access ph }\end{array}$ & & & \\
\hline
\end{tabular}

Fig. 1. Proposed MAC Superframe (SF) for WBAN.

Fig. 1 illustrated the proposed superframe for the delaytolerant MAC protocol which is based on the synchronous mode of IEEE 802.15.6 standard. In our research, we combine EAP1 and EAP2 into EAP for emergency data transmission. In IEEE806.15.6 standard, user priority $\mathrm{UP}_{7}$ defines the priority of the highest level over medical data of different classes including emergency and non-emergency medical data. Besides, in our proposed MAC mechanism, a combined version of Random Access Phase-RAP1 and RAP2 which is named as RAP is proposed for on-demand medical data. In addition, a combined version of Managed Access Phase-MAP1 and MAP2 which is named as MAP is suggested for normal medical or non-medical traffic. Normal medical or nonmedical data is scheduled based whereas; emergency data requires random access in WBANs transmission medium.

In our research, we propose CSMA/CA mechanism for Extensive Access Phase-EAP along with preemptive method over non-preemptive method for contention-free data transmission. EAP is a combination of two contention access phases such as EAP 1, and EAP 2 has been proposed to deploy only for high priority traffic (emergency packets). On the 
contrary, the RAP phase which is the addition of two random access phases RAP 1 and RAP 2 is proposed for on-demand traffic having the average user priority and MAP phase is assigned to the normal medical or non-medical traffics for the traffics with low user priority.

In this research, we deploy six different sensors for measuring different physiological parameters of patients. Physiological parameters are then classified based on data classification concepts of IEEE802.15.6 standard. The emergency data has the highest priority followed by medium priority for on-demand data and the lowest priority for normal medical data. For prioritizing the emergency data we define and classify the medical physiological data on the basis of severity level. According to our study [19-20], data severity level is defined based on the patients' symptoms, mortality rate and hospital admission at overcrowded ritual sites. Emergency data are non-periodic and random in nature and can be generated at any time. Besides, normal medical or non-medical data can be observed periodically. Moreover, using the data threshold values the emergency data and normal medical data can be differentiated easily. Therefore, the priority-severity index value of WBAN traffic is formulated by combining the highest user priority $\left(\mathrm{P}_{7}\right.$ : emergency data) and the index of different data severity level $\left(\mathrm{S}_{1}-\mathrm{S}_{6}\right)$. The priority-severity level is defined using the formula $\mathrm{i}=\mathrm{P}_{7} \mathrm{~S}_{\mathrm{i}}$ where $\mathrm{i}=1-6$, that ranges from $\mathrm{P}_{7} \mathrm{~S}_{1}-\mathrm{P}_{1} \mathrm{~S}_{6}$ with WBANs user priority $\mathrm{UP}_{7}$ followed by $\mathrm{UP}_{6}$ for on-demand traffic $\left(\mathrm{P}_{6}\right.$ : on-demand traffic) and $\mathrm{UP}_{5}$ for normal traffic $\left(\mathrm{P}_{5}\right.$ : normal traffic).

In WBAN communication, body sensors are supposed to sense or generate data, process data according to the methods applied and transmit data to body coordinator (Hub). In contrary, WBAN Hub or body coordinator collects data from sensors; processes and sorts data according to the methods and techniques applied; and then transmit data using appropriate transmission channel of MAC superframe (SF) structure for further processing. Table II presents the projected severity and priority index values.

TABLE II. SEVERITY-PRIORITY INDEX VALUE

\begin{tabular}{|c|c|c|c|}
\hline $\begin{array}{l}\text { WBAN UP } \\
\text { as of IEEE } \\
\mathbf{8 0 6 . 1 5 . 6}\end{array}$ & $\begin{array}{l}\text { Severity Level } \\
\text { of Emergency } \\
\text { Traffic }\end{array}$ & $\begin{array}{l}\text { Criticality- } \\
\text { Priority Index } \\
\text { Table }\end{array}$ & $\begin{array}{l}\text { Types of Access } \\
\text { Phases }\end{array}$ \\
\hline $\begin{array}{l}\mathrm{UP}_{7}=\mathrm{P}_{7}, \\
\text { indicates } \\
\text { Emergency } \\
\text { Medical } \\
\text { Condition }\end{array}$ & $\mathrm{S}_{\mathrm{i}}=\{1,2,3,4,5,6\}$ & $\begin{array}{l}\text { Various } \\
\text { Emergency Data } \\
\text { Types: } \\
\mathrm{P}_{7} \mathrm{~S}_{1}=\left\{\mathrm{P}_{7} \mathrm{~S}_{1}, \mathrm{P}_{7} \mathrm{~S}_{2},\right. \\
\mathrm{P}_{7} \mathrm{~S}_{3}, \mathrm{P}_{7} \mathrm{~S}_{4}, \mathrm{P}_{7} \mathrm{~S}_{5} \\
\left.\mathrm{P}_{7} \mathrm{~S}_{6}\right\}\end{array}$ & $\begin{array}{l}\text { Exclusive Access } \\
\text { Phase EAP that } \\
\text { combines EAP1 } \\
\text { and EAP2 }\end{array}$ \\
\hline $\begin{array}{l}\mathrm{UP}_{6}=\mathrm{P}_{6}, \\
\text { indicates } \\
\text { High Priority } \\
\text { Medical Data }\end{array}$ & $\mathrm{P}_{6} \mathrm{~S}_{\mathrm{i}}=0$ & & $\begin{array}{l}\text { Random Access } \\
\text { Phase RAP that } \\
\text { combines RAP1 } \\
\text { and RAP2 }\end{array}$ \\
\hline $\begin{array}{l}\mathrm{UP}_{5}=\mathrm{P}_{5}, \\
\text { indicates } \\
\text { Medical Data }\end{array}$ & $\mathrm{P}_{5} \mathrm{~S}_{\mathrm{i}}=0$ & & $\begin{array}{l}\text { Managed Access } \\
\text { Phase MAP that } \\
\text { combines MAP1 } \\
\text { and MAP2 }\end{array}$ \\
\hline
\end{tabular}

\section{The Roles of Sensor Nodes and Body Coordinator}

In our proposed delay-tolerant MAC protocol, body coordinator allocates data transmission slot based on user priority levels which rely on different data types, criticality level of emergency data, and priority-severity index value as discussed earlier. Priority levels of different data values are generated by the nodes, except the on-demand data defined by the coordinator. Moreover, criticality levels of emergency data and non-critical normal medical data are specified by the coordinator. The coordinator also determines the prioritycriticality index table, and then slots are assigned accordingly for transmitting data to the healthcare stations. In the proposed delay-tolerant MAC protocol we consider M/M/1 technique with both preemptive and non-preemptive methods where, traffics arrivals are determined by Poisson process. In this research, we also consider diverse user priority $\left(\mathrm{UP}_{7}-\mathrm{UP}_{5}\right)$ and dissimilar severity level of medical emergency data.

In general, a body coordinator receives data, processes it and then transmits the data for further processing on the basis of the priority-severity level. In WBANs operations, multiple emergency events may occur at any moment of time; thus, specifying the roles of each node which is either sensor or hub is urgent to schedule according to the level of criticality and priority. At body coordinator level, an emergency traffic is classified based on data received from the sensors according to the priority severity index values. Hence, upon receiving the packets from physiological sensors, the hub is to use its severity index values to classify the emergency data and express the following resolutions or determinations:

1) If any emergency data exceeds the threshold value as compared to the severity-priority index values, then the status of that precise data will be defined as severe or critical.

2) Moreover, if any data derived from the sensor node (let's consider as on-demand traffic) categorised as on-demand traffic and no corresponding data or type is found in the severity-priority index values, then the health status will be designated as non-critical but an emergency. Also, since the related traffic is designated as an emergency, the coordinator, if required, may include a replica of the data related to the new findings to its severitypriority index table for improvement of the severity index before transmitting it.

3) Finally, if data traffic comes from the sensor or source node, which is categorised as normal, then the pilgrims' health status will be nominated normal medical.

The following Fig. 2 presents the functions of WBAN hub to determine heterogeneous physiological data, data classification, and delay-tolerant channel access mechanism. Moreover, we explain the severity level of data as presented in Fig. 3.

1) The traffic is called as extremely severe traffic, if the severity-priority index value $\mathrm{P}_{7} \mathrm{~S}_{\mathrm{i}}=\mathrm{P}_{7} \mathrm{~S}_{1}$ (the highest severity level of emergency data).

2) The traffic is very high severe traffic, if the severitypriority index value $\mathrm{P}_{7} \mathrm{~S}_{\mathrm{i}}=\mathrm{P}_{7} \mathrm{~S}_{2}$. 
3) The traffic is high severe traffic, if the severity-priority index value $\mathrm{P}_{7} \mathrm{~S}_{\mathrm{i}}=\mathrm{P}_{7} \mathrm{~S}_{3}$.

4) The traffic moderate severe traffic, if the severitypriority index value $\mathrm{P}_{7} \mathrm{~S}_{\mathrm{i}}=\mathrm{P}_{7} \mathrm{~S}_{4}$.

5) The traffic is low severe traffic, if the severity-priority index value $\mathrm{P}_{7} \mathrm{~S}_{\mathrm{i}}=\mathrm{P}_{7} \mathrm{~S}_{5}$.

6) The traffic is very low severe traffic, if the severitypriority index value $\mathrm{P}_{7} \mathrm{~S}_{\mathrm{i}}=\mathrm{P}_{7} \mathrm{~S}_{6}$.

\section{Relevant Algorithms and Slot Allocation in Modified MAC Superframe Structure}

A slot allocation mechanism for MAC superframe has been proposed by the type of data according to threshold values from body sensor nodes and order of criticality level of both emergency and non-emergency data traffic form body coordinator node at different pilgrims' health conditions. For this, emergency traffic is categorised into six types based on the criticality level of vital signs as discussed earlier.

Pseudo code: Heterogeneous physiological data determination, classification and delay-tolerant channel access mechanism

Input: Threshold parameter of sensed physiological data

Sensing Element: Various sensors

Output: Classification of medical data, access to communication channel

\section{Begin}

//Patients' medical data received by Sensors and coordinate to Hub for further processing

1. if data exceeds $(>$ or $<$ ) threshold limit then data is classified as emergency medical data; Set the priority level UP7;

Define the delay-tolerant preemptive or non preemptive methods for lower delay communication;

EAP of SF is selected to transfer data and access the channel using CSMA/CA mechanism;

2. elseif data exceeds $(>$ or $<)$ or within $(<>)$ threshold limit then data is classified as ondemand medical data;

Set the priority level UP6;

Data to be transmitted as requested by healthcare station;

RAP of SF is selected to transfer data and access the channel using CSMA/CA mechanism;

3. elseif data within $(\diamond)$ threshold value then data is classified as normal medical data;

Set the priority level UP5;

Data is transmitted using scheduled access mechanism;

MAP of SF is selected to transfer data
4. else
Repeat step 1

5. end if

End

Fig. 2. Identification of Heterogeneous Traffic.
Pseudo code: Determine the severity level of emergency medical data at WBSN Hub

Input:

T: Traffic or patients' physiological data

$\mathrm{P}_{1} \mathrm{C}_{\mathrm{i}}$ : Severity-priority index value

Output:

Emergency events classification based on data severity level Begin

// for every arrive data from the sensor node

1. if (data type $==$ emergency) $\& \&\left(\mathrm{P}_{7} \mathrm{~S}_{\mathrm{i}}==\mathrm{P}_{7} \mathrm{~S}_{1}\right)$ then

$\mathrm{T}$ nominates as extremely severe traffic

2. elseif (data type $==$ emergency) \&\& $\left(\mathrm{P}_{7} \mathrm{~S}_{\mathrm{i}}==\mathrm{P}_{7} \mathrm{~S}_{2}\right)$ then

$\mathrm{T}$ nominates as very high severe traffic

3. elseif (data type $==$ emergency) \&\& $\left(\mathrm{P}_{7} \mathrm{~S}_{\mathrm{i}}==\mathrm{P}_{7} \mathrm{~S}_{3}\right)$ then

$\mathrm{T}$ nominates as high severe traffic

4. elseif (data type $==$ emergency) \&\& $\left(\mathrm{P}_{7} \mathrm{~S}_{\mathrm{i}}==\mathrm{P}_{7} \mathrm{~S}_{4}\right)$ then

$\mathrm{T}$ nominates as moderately severe traffic

5. elseif (data type $==$ emergency) \& \& $\left(\mathrm{P}_{7} \mathrm{~S}_{\mathrm{i}}==\mathrm{P}_{7} \mathrm{~S}_{5}\right)$ then

$\mathrm{T}$ nominates as low severe traffic

6. elseif (data type $==$ emergency) \& \& $\left(\mathrm{P}_{7} \mathrm{~S}_{\mathrm{i}}==\mathrm{P}_{7} \mathrm{~S}_{6}\right)$ then

$\mathrm{T}$ nominates as very low severe traffic

7. else

if (data type $==$ on-demand $) \& \&\left(P_{7} S_{i}==0\right)$ then

$\mathrm{T}$ nominates as on-demand

14. end if

15. else

16. if (data type $==$ no event or normal) \&\&( $\left.P_{7} S_{i}==0\right)$ then

$\mathrm{T}$ nominates as normal traffic

17. end if

18. end if

19. end

Fig. 3. Body Coordinator's Role to Deal with Emergency Traffic

If the coordinator at any moment of time receives emergency data from a sensor, then it assigns EAP slot to this particular traffic based on the CSMA/CA mechanism. Here there is no need for finding the criticality level of that emergency traffic because it has been received from the single sensor node.

When two or more than two emergency traffics are received by the coordinator at a time, it invokes the severitypriority index value as illustrated earlier in Table II. Upon classification phase, depending on the traffic types, data are to be distributed among the queues. All emergency data shall proceed to slot EAP based on their criticality level and queuing process. Traffic queuing process at the data transmission scheme of the MAC level is illustrated in Fig. 4. Emergency traffic is life-threatening, so excessive delay and data loss can worsen the health condition or life of the pilgrims. Hence it is vital to ensure no data loss and minimal delay in emergency traffic.

In our research, we assume, on-demand and normal traffic are not life-threatening. RAP phase is allocated for on-demand traffics which are contention-based, since this traffic is considered as not life-threatening so low to moderate contention for the slots are acceptable when there is multiple such traffics. For normal traffic, a scheduled access phase MAP is assigned so that data traffic can access the channel at a specific period. Again, since this traffic is assumed as not lifethreatening so low to moderate contention and delay for the slots are acceptable when there is multiple such traffics. The queuing process for emergency data transmission is presented in Fig. 4. 


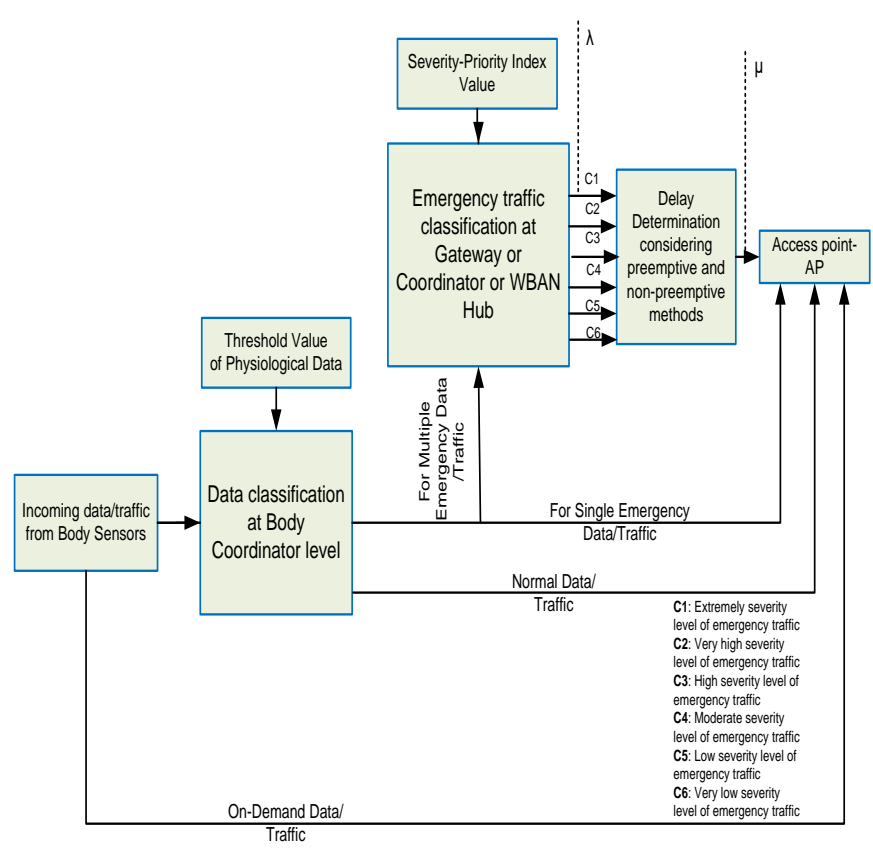

Fig. 4. Queuing Process of Emergency Data Transmission through MAC Protocol.

Preemptive method is time-critical and applicable for emergency traffic which is random in nature. In addition, preemptive method allows data with the highest precedence access the communication channel ahead of data with lower precedence. The preemption technique is illustrated in Fig. 5.

Non-preemptive method is non-time-critical and applicable for normal medical traffic and even can be utilized for the emergency traffic with different severity levels in WBAN communication. Normal medical traffic can be of time bounded or scheduled in nature. The non-preemption technique is illustrated in Fig. 6.

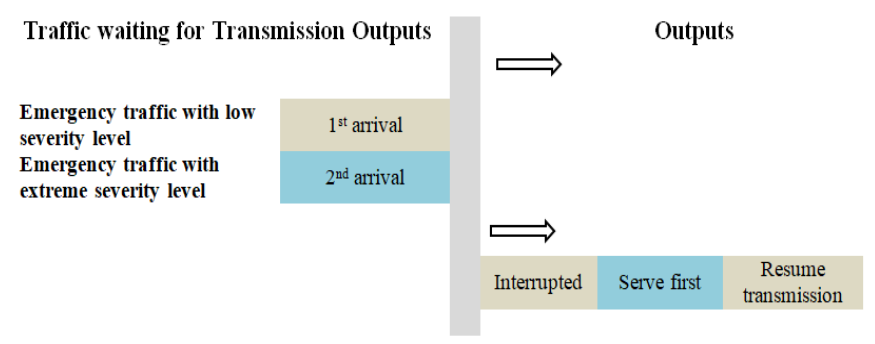

Fig. 5. Schematic Diagram of Preemption.

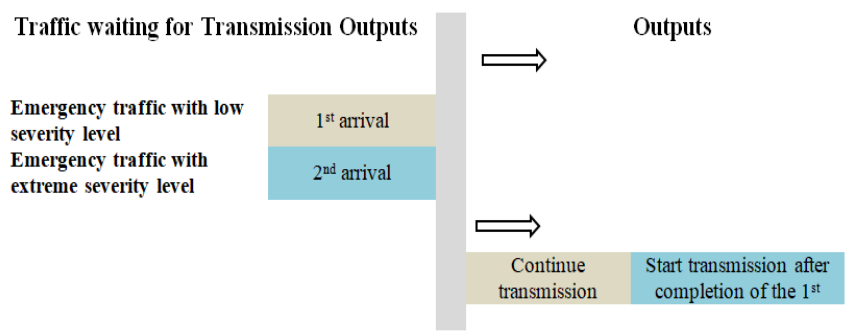

Fig. 6. Schematic Diagram of non-preemption.
For the proposed delay-tolerant MAC protocol we deploy CSMA/CA mechanism for handling emergency traffic of WBAN. Since the proposed MAC is supposed to transmit emergency traffic with different severity levels hence, the length of backoff counter (BC) should be set to minimal and data transmission is occurred when $\mathrm{BC}=0$ which is presented in Fig 7. In addition, the length of Contention Window (CW) of MAC superframe (SF) is varied according to the type of applications. In our research, $\mathrm{CW}$ is fixed to minimum as $\mathrm{CW}=\mathrm{CW}_{\text {min }}$ for emergency data transmission, however, for non-emergency cases the $\mathrm{CW}$ is set to $\mathrm{CW}_{\max }$.

\section{E. Mathematical Analysis of the Proposed Delay-tolerant MAC Protocol Considering Preemptive Method}

In our research, the queuing delay is measured using the deployment of various queue methods as explained earlier in this research paper. The queue model has been proposed in our research demonstrates service time as queuing delay that is being served in the system. The delay is calculated as follows, where; $\lambda$ is data or traffic arrival rate, $\mu$ is the service rate of traffic, traffic intensity rate is denoted by $\rho$, according to our research, $\mathrm{E}(n)$ is the average number of critical data in the queue, $\mathrm{E}(\mathrm{t})$ is average or mean delay time for any kind of traffic or data.

Extremely severe traffic: $\mathrm{E}\left(t_{\mathrm{p} 7 \mathrm{~s} 1}\right)=\frac{\frac{1}{\mu}}{1-\rho_{\mathrm{p} 7 \mathrm{~s} 1}}$

Very high severe traffic: $\mathrm{E}\left(t_{\mathrm{p} 7 \mathrm{~s} 2}\right)=\frac{\mathrm{E}\left(\mathrm{n}_{\mathrm{p} 7 \mathrm{~s} 2}\right)}{\lambda \mathrm{p} 7 \mathrm{~s} 2}=$

$\frac{\frac{1}{\mu}}{\left(1-\rho_{\mathrm{p} 7 \mathrm{~s} 1}\right)\left(1-\rho_{\mathrm{p} 7 \mathrm{~s} 1}-\rho_{\mathrm{p} 7 \mathrm{~s} 2}\right)}$

High severe traffic: $\mathrm{E}\left(t_{\mathrm{p} 7 \mathrm{~s} 3}\right)=\frac{\mathrm{E}\left(\mathrm{n}_{\mathrm{p} 7 \mathrm{~s} 3}\right)}{\lambda \mathrm{p} 7 \mathrm{~s} 3}=$

$\frac{\frac{1}{\mu}}{\left(1-\rho_{\mathrm{p} 7 \mathrm{~s} 1}-\rho_{\mathrm{p} 7 \mathrm{~s} 2}\right)\left(1-\rho_{\mathrm{p} 7 \mathrm{~s} 1}-\rho_{\mathrm{p} 7 \mathrm{~s} 2}-\rho_{\mathrm{p} 7 \mathrm{~s} 3}\right)}$

Moderately severe traffic: $\mathrm{E}\left(t_{\mathrm{p} 7 \mathrm{~s} 4}\right)=\frac{\mathrm{E}\left(\mathrm{n}_{\mathrm{p} 7 \mathrm{~s} 4}\right)}{\lambda \mathrm{p} 7 \mathrm{~s} 4}=$

$\frac{\frac{1}{\mu}}{\left(1-\rho_{\mathrm{p} 7 \mathrm{~s} 1}-\rho_{\mathrm{p} 7 \mathrm{~s} 2}-\rho_{\mathrm{p} 7 \mathrm{~s} 3}\right)\left(1-\rho_{\mathrm{p} 7 \mathrm{~s} 1}-\rho_{\mathrm{p} 7 \mathrm{~s} 2}-\rho_{\mathrm{p} 7 \mathrm{~s} 3}-\rho_{\mathrm{p} 7 \mathrm{~s} 4}\right)}$

Low severe traffic: $\mathrm{E}\left(t_{\mathrm{p} 7 \mathrm{s5}}\right)=\frac{\mathrm{E}\left(\mathrm{n}_{\mathrm{p} 7 \mathrm{s5}}\right)}{\lambda \mathrm{p} 7 \mathrm{~s} 5}=$

$\frac{\frac{1}{\mu}}{\left(1-\rho_{\mathrm{p} 7 \mathrm{~s} 1}-\rho_{\mathrm{p} 7 \mathrm{~s} 2}-\rho_{\mathrm{p} 7 \mathrm{~s} 3}-\rho_{\mathrm{p} 7 \mathrm{~s} 4}\right)\left(1-\rho_{\mathrm{p} 7 \mathrm{~s} 1}-\rho_{\mathrm{p} 7 \mathrm{~s} 2}-\rho_{\mathrm{p} 7 \mathrm{~s} 3}-\rho_{\mathrm{p} 7 \mathrm{~s} 4}-\rho_{\mathrm{p} 7 \mathrm{~s} 5}\right)}$

Very low severe traffic: $\mathrm{E}\left(t_{\mathrm{p} 7 \mathrm{~s} 6}\right)=\frac{\mathrm{E}\left(\mathrm{n}_{\mathrm{p} 7 \mathrm{~s} 6}\right)}{\lambda \mathrm{p} 7 \mathrm{~s} 6}=$

$\frac{\frac{1}{\mu}}{\left(1-\rho_{\mathrm{p} 7 \mathrm{~s} 1}-\rho_{\mathrm{p} 7 \mathrm{~s} 2}-\rho_{\mathrm{p} 7 \mathrm{~s} 3}-\rho_{\mathrm{p} 7 \mathrm{~s} 4}-\rho_{\mathrm{p} 7 \mathrm{~s} 5}\right)\left(1-\rho_{\mathrm{p} 7 \mathrm{~s} 1}-\rho_{\mathrm{p} 7 \mathrm{~s} 2}-\rho_{\mathrm{p} 7 \mathrm{~s} 3}-\rho_{\mathrm{p} 7 \mathrm{~s} 4}-\rho_{\mathrm{p} 7 \mathrm{~s} 5}\right)}$

And applying Little's law of Queuing theory we get,

$E\left(t_{\mathrm{p} 7 \mathrm{~s}}\right)=\frac{E\left(n_{p 7 s i}\right)}{\lambda p 7 s i}=\frac{\frac{1}{\mu}}{\left(1-\left(\sum_{i=1}^{n-1} \rho_{p 7 s_{i}}\right)\right)\left(1-\left(\sum_{i=1}^{n} \rho_{p 7 s_{i}}\right)\right)}$ 


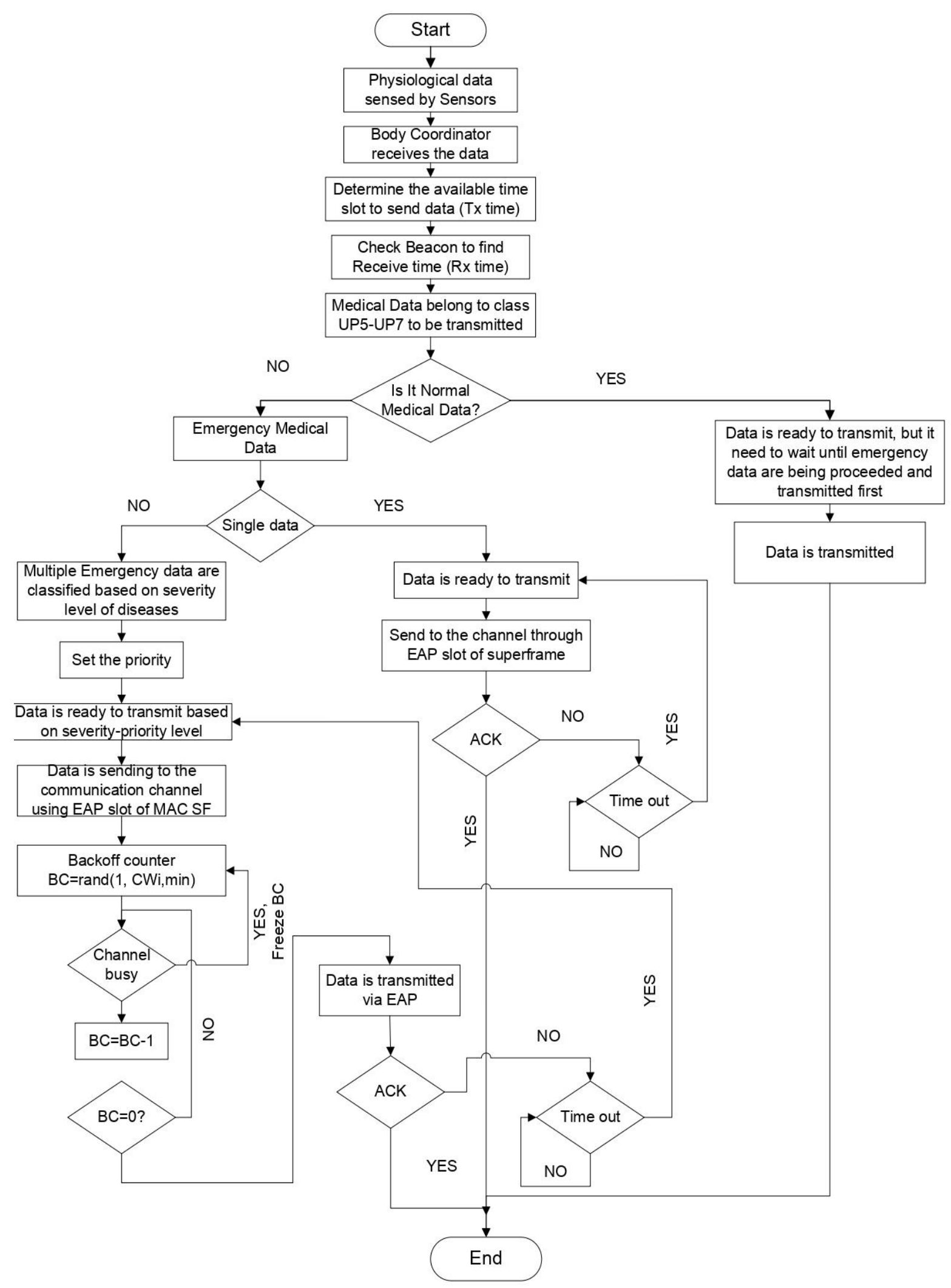

Fig. 7. CSMA/CA Mechanism and Relevant Operational Flowchart of the Proposed Delay-tolerant MAC Protocol. 


\section{F. Mathematical Analysis of the Proposed Delay-tolerant MAC Protocol Considering Non-Preemptive Method}

In fact, by introducing Little's law in our research work and the property of PASTA (Poisson Arrivals See Time Averages), $\mathrm{E}\left(n_{\mathrm{plc1}}\right)$ and $E\left(t_{\mathrm{plc1}}\right)$ can be defined directly without expressing the probabilities $\mathrm{P}_{\mathrm{n}}$. According to PASTA, in the system, an average number of customers seen by an arriving customer equals $\mathrm{E}\left(n_{\mathrm{p} 7 \mathrm{~s} 1}\right)$ and each of the customers has a service time (residual) with mean $\frac{1}{\mu}$. Additionally, patient must wait for its own service time. Hence, the average or mean time for extremely severe data can be formulated as, where the queue delay is determined considering the packets in service plus the packets that are already buffered in the queue.

$\mathrm{E}\left(t_{\mathrm{p} 7 \mathrm{~s} 1}\right)=\frac{\mathrm{E}\left(\mathrm{n}_{\mathrm{p} 7 \mathrm{~s} 1}\right)}{\mu}+\frac{1}{\mu}+\frac{1}{\mu}\left(\rho_{\mathrm{p} 7 \mathrm{~s} 2}+\rho_{\mathrm{p} 7 \mathrm{~s} 3}+\rho_{\mathrm{p} 7 \mathrm{~s} 4}+\rho_{\mathrm{p} 7 \mathrm{~s} 5}+\right.$

$\left.\rho_{\mathrm{p} 7 \mathrm{~s} 6}\right)$

$\mathrm{E}\left(t_{\mathrm{p} 7 \mathrm{~s} 1}\right)=\frac{\mathrm{E}\left(\mathrm{n}_{\mathrm{p} 7 \mathrm{~s} 1}\right)}{\mu}+\frac{1}{\mu}+\frac{1}{\mu}\left(\rho-\rho_{\mathrm{p} 7 \mathrm{~s} 1}\right)$

Very high severe data processing: The very high severe data packet has to wait for the extremely severe data in service and very high severe data or packets in the queue. The delay can be found using the following formula:

$$
\begin{aligned}
& \mathrm{E}\left(t_{\mathrm{p} 7 \mathrm{~s} 2}\right)=\frac{E\left(n_{p 7 s 1}\right)}{\mu}+\frac{1}{\mu}+\frac{1}{\mu}\left(\rho_{p 7 s 3}+\rho_{p 7 s 4}+\rho_{p 7 s 5}+\rho_{p 7 s 6}\right) \\
& \mathrm{E}\left(t_{\mathrm{p} 7 \mathrm{~s} 2}\right)=\frac{E\left(n_{p 7 s 1}\right)}{\mu}+\frac{1}{\mu}+\frac{1}{\mu}\left(\rho-\rho_{p 7 s 1}-\rho_{p 7 s 2}\right)
\end{aligned}
$$

High severe data processing: The high severe packet has to wait for the extremely severe packet and very high severe packets in service and high severe data or packets in the queue. The delay can be found using the following formula:

$$
\begin{aligned}
& \mathrm{E}\left(t_{\mathrm{p} 7 \mathrm{~s} 3}\right)=\frac{E\left(n_{p 7 s 1}\right)}{\mu}+\frac{E\left(n_{p 7 s 2}\right)}{\mu}+\frac{1}{\mu}+\frac{1}{\mu}\left(\rho_{p 7 s 4}+\rho_{p 7 s 5}+\rho_{p 7 s 6}\right) \\
& \mathrm{E}(t \mathrm{p} 7 \mathrm{~s} 3)=\frac{E\left(n_{p 7 s 1}\right)}{\mu}+\frac{E\left(n_{p 7 s 2}\right)}{\mu}+\frac{1}{\mu}+\frac{1}{\mu}\left(\rho-\rho_{p 7 s 1}-\rho_{p 7 s 2}-\right. \\
& \left.\rho_{p 7 s 3}\right)
\end{aligned}
$$

Moderately severe data processing: The moderate severe data has to wait for the extremely severe packet, very high severe packet and high severe packet in service and moderate severe da ta or packets in the queue. The delay can be found using the following formula:

$$
\begin{aligned}
& \mathrm{E}\left(t_{\mathrm{p} 7 s 4}\right)=\frac{E\left(n_{p 7 s 1}\right)}{\mu}+\frac{E\left(n_{p 7 s 2}\right)}{\mu}+\frac{E\left(n_{p 7 s 3}\right)}{\mu}+\frac{1}{\mu}+\frac{1}{\mu}\left(\rho_{p 7 s 5}+\rho_{p 7 s 6}\right) \\
& \mathrm{E}\left(t_{\mathrm{p} 7 s 4}\right)=\frac{E\left(n_{p 7 s 1}\right)}{\mu}+\frac{E\left(n_{p 7 s 2}\right)}{\mu}+\frac{E\left(n_{p 7 s 3}\right)}{\mu}+\frac{1}{\mu}+\frac{1}{\mu}\left(\rho-\rho_{p 1 c 1}-\right. \\
& \left.\rho_{p 1 c 2}-\rho_{p 1 c 3}-\rho_{p 1 c 4}\right)
\end{aligned}
$$

Low severe data processing: The low severe data has to wait for the extremely severe packet, very high severe packet, high severe packet and moderate severe data in service and low severe data or packets in the queue. The delay can be found using the following formula:

$$
\begin{aligned}
& \mathrm{E}(\mathrm{tp} 7 \mathrm{~s} 5)=\frac{E\left(n_{p 7 s 1}\right)}{\mu}+\frac{E\left(n_{p 7 s 2}\right)}{\mu}+\frac{E\left(n_{p 7 s 3}\right)}{\mu}+\frac{E\left(n_{p 7 s 4}\right)}{\mu}+\frac{1}{\mu}+ \\
& \frac{1}{\mu}\left(\rho-\rho_{p 7 s 1}-\rho_{p 7 s 2}-\rho_{p 7 s 4}-\rho_{p 7 s 4}-\rho_{p 7 s 5}\right)
\end{aligned}
$$

$$
\mathrm{E}(t \mathrm{p} 7 \mathrm{~s} 5)=\frac{E\left(n_{p 7 s 1}\right)}{\mu}+\frac{E\left(n_{p 7 s 2}\right)}{\mu}+\frac{E\left(n_{p 7 s 3}\right)}{\mu}+\frac{E\left(n_{p 7 s 4}\right)}{\mu}+\frac{1}{\mu}\left(\rho_{p 7 s 6}\right)
$$

Very low severe data processing: The low severe data has to wait for the extremely severe packet, very high severe packet, high severe packet, moderate severe data and low severe data in service and very low severe data or packets in the queue. The delay can be found using the following formula:

$$
\begin{aligned}
& \mathrm{E}(t \mathrm{p} 7 \mathrm{~s} 6)=\frac{E\left(n_{p 7 s 1}\right)}{\mu}+\frac{E\left(n_{p 7 s 2}\right)}{\mu}+\frac{E\left(n_{p 7 s 3}\right)}{\mu}+\frac{E\left(n_{p 7 s 4}\right)}{\mu}+ \\
& \frac{E\left(n_{p 7 s 5}\right)}{\mu}+\frac{1}{\mu}+\frac{1}{\mu}\left(\rho-\rho_{p 7 s 1}-\rho_{p 7 s 2}-\rho_{p 7 s 4}-\rho_{p 7 s 4}-\rho_{p 7 s 5}-\right. \\
& \left.\rho_{p 7 s 6}\right)
\end{aligned}
$$

$\mathrm{E}(t \mathrm{p} 7 \mathrm{~s} 6)=\frac{E\left(n_{p 7 s 1}\right)}{\mu}+\frac{E\left(n_{p 7 s 2}\right)}{\mu}+\frac{E\left(n_{p 7 s 3}\right)}{\mu}+\frac{E\left(n_{p 7 s 4}\right)}{\mu}+$ $\frac{E\left(n_{p 7 s 4}\right)}{\mu}+\frac{1}{\mu}$

\section{RESULT AND DISCUSSION}

\section{A. Simulation Environment}

The proposed delay-tolerant MAC protocol has been experimented and simulated using Castalia simulator which is based on OMNeT++ platform. Castalia is built for network of low-power embedded devices.

\section{B. Simulation Parameters}

To evaluate the competency of delay-tolerant MAC protocol, different scenarios are being considered including network size (number of nodes) and diverse traffic size. Varied network size has been considered ranging from 1 to 6 sensor nodes. In addition, different traffic sizes have been taken ranging from 16 bytes to 127 bytes. WBAN operating range is considered ranging from 5 to 10 meters with operational frequency of $2.4 \mathrm{GHz}$ and channel bandwidth is $250 \mathrm{kbps}$. Simulation parameters are depicted in subsequent Table III.

TABLE III. MAC SIMULATION PARAMETERS

\begin{tabular}{|l|l|}
\hline Parameters & Values \\
\hline Total Nodes & 6 \\
\hline Body Coordinator & 1 \\
\hline Data Transmission Range & $3 \mathrm{~m}$ to $5 \mathrm{~m}$ \\
\hline mMaxBANSize & $<64$ Nodes \\
\hline MAC and Superframe Structure & IEEE 802.15 .6 Standard \\
\hline Channel Mode & Wireless \\
\hline MAC Superframe Size & Total 255 Slots \\
\hline MAC Superframe Duration & 122.88 ms \\
\hline Simulation Runtime & 150 Seconds \\
\hline Operating Frequency & 2.4 GHz ISM \\
\hline Bandwidth & Up to 250 kbps \\
\hline Packet Size & Variable, up to 512 bytes \\
\hline macMaxCSMABackoffs & $*$ So far there is no specific unit \\
\hline Beacon size & 40 bytes \\
\hline
\end{tabular}




\section{Performance Evaluation and Results Discussion}

In order to performance evaluation of the proposed delaytolerant MAC protocol, different scenarios are considered including network size (number of sensor nodes) and diverse traffic size. In our proposed MAC model we assume WBAN with a star topology. We also set EAP1, EAP2, RAP1, RAP2 and CAP to zero for the proposed MAC superframe (SF) structure and set to EAP, RAP and MAP for diverse traffics. The proposed delay-tolerant MAC protocol is compared considering both preemptive and non-preemptive methods for analyzing the performance. Moreover, the delay is compared with Traffic-aware MAC (TA-MAC) protocol for further validation of the proposed scheme.

\section{First Scenario}

The first scenario portrays the delay comparison considering both preemptive and non-preemptive methods considering varying nodes. For analysis delay we considered heterogeneous emergency traffic with different levels of severity. Fig. 8 presents the WBAN situation where the total number of nodes or network size increases from 1 to 6 . From the obtained result it has been seen that the queue delay considering preemptive and non-preemptive methods for different emergency data increases with the increasing network size. In general, if the network size or number of nodes increased in the network then the delay is also increased proportionally almost in all the cases as depicted in Fig. 8. Moreover, the proposed MAC protocol performs better in term of delay by applying preemptive method in the network than that of non-premptive method.

In non-preemptive method, the delay is increased for the transmission of emergency traffics because traffic with higher severity level has to wait until traffic with lower severity level complete its transmission which is already being served. However, the proposed delay-tolerant MAC works better with preemptive method because in this method emergency traffic with higher severity levels proceed ahead of normal medical data or emergency data with lower severity level. Form the experiment it is obtained that WBAN with single node and preemptive method takes $35.87 \mathrm{~ms}$ whereas the same network model with non-preemptive method takes almost $50 \mathrm{~ms}$ for data transmission using the modified MAC superframe and required EAP channel. Moreover, the delay is increased in all the cases if the nodes number is also increased using both queue methods. From our experiment it is found that WBAN with six nodes and preemptive method takes almost $58.50 \mathrm{~ms}$ whereas the same network model with non-preemptive method takes almost $72 \mathrm{~ms}$ for data transmission which is much higher than that of the preemptive method.

\section{E. Second Scenario}

The second scenario analyses the delay efficiency of proposed MAC by considering different severity levels of emergency traffic. The results are obtained on the basis of different traffic sizes which are fluctuating from 16 bytes to 127 bytes.

Fig. 9 shows the obtained delay by implementing preemptive method for our proposed MAC model for different severity levels and the result differs with varied packets (medical) sizes up to 127 bytes. According to the results obtained as presented in Fig. 9, it is shown that for low sized packets (16 bytes) and for extremely severe traffic delay is calculated as $5.78 \mathrm{~ms}$. In contrary, for low sized packets (16 bytes) but for very low severe traffic delay is calculated as 8 ms. It has been observed that the overall delay is increased in the network if the packets sizes up and for 127 bytes packets the delay is measure as $8.12 \mathrm{~ms}$ and $11.89 \mathrm{~ms}$ respectively for extremely severe traffic and very low severe traffic in WBAN communication.

On the other hand, Fig. 10 shows the obtained delay by implementing non-preemptive method for our proposed MAC model for different severity levels with varied packets sizes fluctuating from 16 bytes to 127 bytes. According to the results obtained as presented in Fig. 10, it is shown that for low sized packets (16 bytes) and for extremely severe traffic delay is calculated as $9.3 \mathrm{~ms}$. In contrary, for low sized packets (16 bytes) but for very low severe traffic delay is calculated as 11.1 ms. It has been observed that the overall delay is increased in the network if the packets sizes up and for 127 bytes packets the delay is measure as $11.2 \mathrm{~ms}$ and $14.8 \mathrm{~ms}$ respectively for extremely severe traffic and very low severe traffic in WBAN communication.

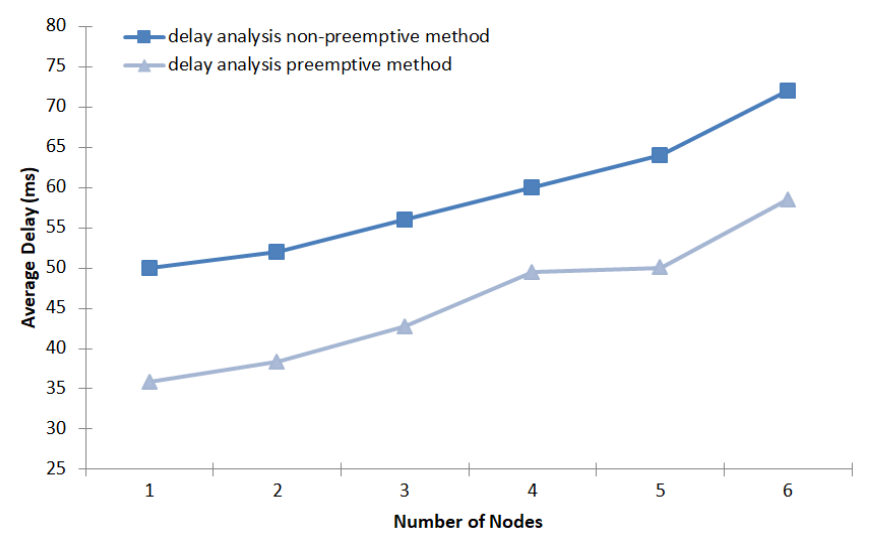

Fig. 8. Delay Comparison of Emergency Data Transmission Considering Preemptive and Non-preemptive Methods.

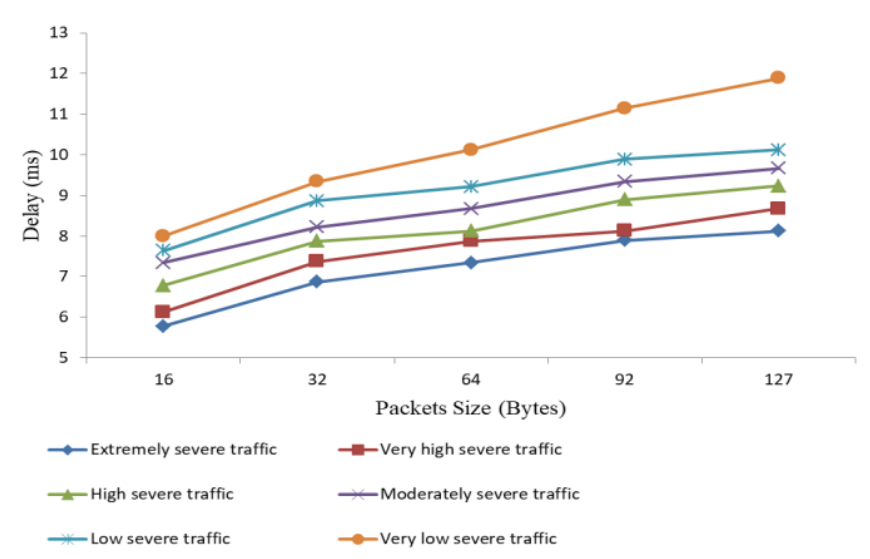

Fig. 9. Delay Assessment of Emergency Traffic Considering Preemptive Method. 


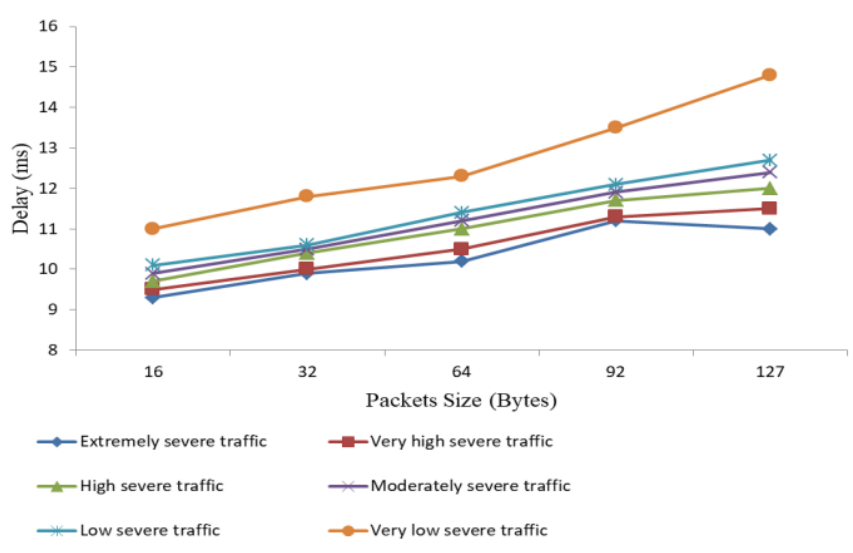

Fig. 10. Delay Assessment of Emergency Traffic Considering NonPreemptive Method.

Moreover, our proposed delay-tolerant MAC protocol with both queue methods has been compared with up-to-date Traffic-aware (TA-MAC) protocol and it has been found that our proposed model with preemptive method preforms much better than TA-MAC in terms of delay in data transmission in WBAN environment, whereas non-preemptive method based approach consume more time for data transmission as presented in Fig. 11. According the results obtained, it is shown that for low sized packets (16 bytes) and for extremely severe traffic delay is calculated as $5.78 \mathrm{~ms}$. In contrary, for low sized packets (16 bytes) but for very low severe traffic delay is calculated as $8 \mathrm{~ms}$ using preemptive method. However, TA-MAC requires $6.3 \mathrm{~ms}$ for extremely severe traffic and 9.11 $\mathrm{ms}$ for very low severe traffic transmission in WBAN.

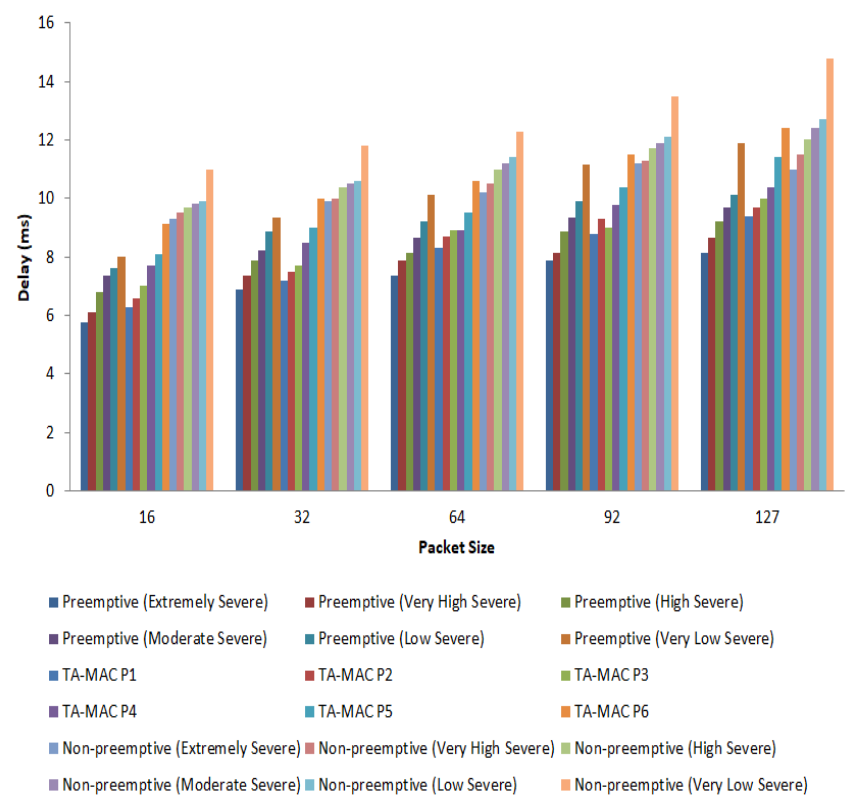

Fig. 11. Delay (ms) is Analysed on the basis of Data Severity Level Considering Various Packet Size.

\section{F. Third Scenario}

The third scenario portrays the delay comparison between the proposed delay-tolerant MAC protocol and TA-MAC protocol. For analysis delay we considered heterogenous emergency traffic with different levels of severity. Fig. 12 presents the WBAN situation where the network size increases from 1 to 6 nodes. Considering 6 as a maximum number of nodes, the queue delay of delay-tolerant MAC protocol is lower than that of competitive TA-MAC protocol.

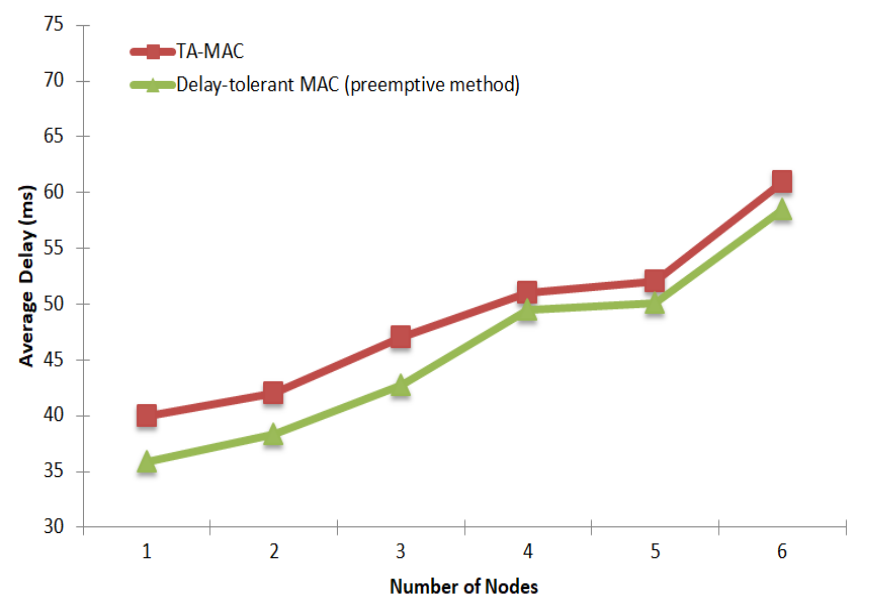

Fig. 12. Delay (ms) Comparison between Proposed MAC and TA-MAC.

\section{CONCLUSION}

In order to facilitate patients' with real-time and quick healthcare services at crowded sites we propose WBANs delay-tolerant MAC protocol. We designed the MAC protocol on the basis of $\mathrm{M} / \mathrm{M} / 1$ preemptive method for dealing with emergency data. However, to analysis the delay efficiency we compare the proposed MAC with both preemptive and nonpreemptive methods. In addition, to design the delay-tolerant MAC for monitoring pilgrims' emergency conditions we categorized emergencies based on level of severity. We proposed an improved MAC superframe structure and algorithms are also developed for better management of sensor nodes and body coordinator at WBANs MAC level. Moreover minimum backoff period and minimum Contention Window $\left(\mathrm{CM}_{\min }\right)$ are considered for quick access to the IEEE 802.15.6 standard based EAP channel. The proposed delay-tolerant MAC protocol has been experimented via simulation and verified using Castalia simulator which is based on OMNeT++ platform. The proposed MAC scheme is designed to handle the emergency situation with different severity levels and during the experiment it has been shown that data transmission using preemptive method works faster with reduced delay than that of non-preemptive method. Furthermore, the proposed delaytolerant MAC protocol is analyzed and compared with up-todate TA-MAC protocol considering its delay matrix. Results demonstrate that delay is relatively low during emergency data transmission in WBANs environment. Our future research plan is to extend the existing work using other queue models including $\mathrm{M} / \mathrm{G} / 1$ and relevant techniques considering WBANs heterogeneous traffics. 


\section{ACKNOWLEDGMENT}

We would like to convey our sincerest appreciation to University of Asia Pacific, Dhaka, Bangladesh for providing us with admirable research environment.

\section{REFERENCES}

[1] Aldossari M, et.al.; Health issues in the Hajj pilgrimage: a literature review. East Mediterr Health J. 2019;25(10):744-753.

[2] Hajj guidelines by Ministry of Health $(\mathrm{MoH})$, Kingdom of Saudi Srabia : https://www.moh.gov.sa/Hajj/Documents/Languages/English.pdf.

[3] Osman, M, "Hajj guide systems - past, present and future", International Journal of Emerging Technology and Advanced Engineering, vol.4, no. 8, pp. 25-31, 2014.

[4] Aladdein Amro, "Pilgrims' Hajj Tracking System," Contemporary Engineering Sciences, Vol. 5, No. 9, pp. 437-446, 2012.

[5] Mohandes, M, et.al., "Pilgrim tracking and identification using wireless sensor networks and GPS in a mobile phone", Arabian Journal for Science and Engineering, vol. 38. No. 8, pp. 2135-2141, 2013.

[6] Memish, Z., "Emergence of medicine for mass gatherings: lessons from the Hajj", The Lancet infectious diseases, vol. 12, no. 1, pp. 56-65, 2012.

[7] Hamhoum, F., "Supporting pilgrims in navigating densely crowded religious sites", Personal and Ubiquitous Computing, vol. 16, no. 8, pp. 1013-1023, 2012.

[8] IEEE. (2012). IEEE Standard for Local and Metropolitan Area Networks - Part 15.6: Wireless Body Area Networks. In IEEE Std 802.15.6-2012. https://doi.org/10.1109/IEEESTD.2012.6161600.

[9] Ghassan Ahmed Ali and Shah Murtaza Rashid Al Masud, "Routing Optimization in WBAN using Bees Algorithm for Overcrowded Haij. Environment" International Journal of Advanced Computer Science and Applications (IJACSA), 9(5), 2018.

[10] Gouda, K. C., et. al., "Implementation of Traffic Priority Aware Medium Access Control Protocol for Wireless Body Area Networks", Springer Link, 1 (7), 2019.
[11] Yuan, D., et.al., “An Adaptive MAC Protocol Based on IEEE802.15.6 for Wireless Body Area Networks", Wireless Communications and Mobile Computing, 1 (9), 2019.

[12] Rismanian Yazdi, et. al., "A Priority-Based MAC Protocol for Energy Consumption and Delay Guaranteed in Wireless Body Area Networks", Wireless Personal Communications, May 2019.

[13] Bhandari, S. et.al., "A MAC Protocol with Dynamic Allocation of Time Slots Based on Traffic Priority in Wireless Body Area Networks", International Journal of Computer Networks \& Communications, 11(4), 25-41, 2019.

[14] Ambigavathi, M., et. al., "Traffic Priority Based Channel Assignment Technique for Critical Data Transmission in Wireless Body Area Network", Journal of Medical Systems, 42(11), 2018.

[15] Sridharan, D., et. al., "Energy efficient and load balanced priority queue algorithm for Wireless Body Area Networks", Future Generation Computer Systems, 88 (February 2019), 586-593.

[16] Rasheed, M. B., et. al., "Delay and energy consumption analysis of priority guaranteed MAC protocol for wireless body area networks", Wireless Networks, 23(4), 1249-1266, 2017.

[17] Henna, S., et. al., "A fair contention access scheme for low-priority traffic in wireless body area networks", Sensors, 17 (9), 2017.

[18] Moh, S. et. al., "A priority-based adaptive MAC protocol for wireless body area networks", Sensors, 16 (3), 2016.

[19] Ansar Munir Shah, Abdelzahir Abdelmaboud, Khalid Mahmood, Mahmood ul Hassan and Muhammad Kashif Saeed, "eHealth WBAN: Energy-Efficient and Priority-Based Enhanced IEEE802.15.6 CSMA/CA MAC Protocol" International Journal of Advanced Computer Science and Applications (IJACSA), 9(4), 2018.

[20] Shah Murtaza Rashid Al Masud, et. al., 'Preemptive Queue Based Modified MAC Superframe for WBSN to Efficiently Transmit Pilgrims' Heterogeneous Data at Ritual Sites: An Analytical Approach'. IJCSNS (International Journal of Computer Science and Network Security), Korea, October 2020 Edition (Vol. 20, No: 10). Indexed in ESCI, Thomson Reuters (ISI). 